\title{
Physical Activities, Sports and Female Pelvic Floor: From Causes to Management-A Review
}

\author{
Alain P Bourcier ${ }^{1 *}$, François Haab ${ }^{2}$ and Jean A Juras ${ }^{3}$ \\ ${ }^{1}$ Centre Imagerie Médicale Cardinet, Urodynamics Unit-Centre Urologie Opéra, \\ Rehabilitation Unit, Paris, France \\ ${ }^{2}$ American Hospital of Paris, Surgery Department-Centre Urologie Opéra, Female \\ Urology, Paris, France \\ ${ }^{3}$ Centre Imagerie Médicale Cardinet, Female Radiology, Paris, France \\ *Corresponding Author: Alain P Bourcier, Centre Imagerie Médicale Cardinet, \\ Urodynamics Unit-Centre Urologie Opéra, Rehabilitation Unit, Paris, France.
}

\author{
Received: April 20, 2020 \\ Published: May 12, 2020 \\ (C) All rights are reserved by Rene \\ Gonzalez-Fernandez., et al.
}

\begin{abstract}
There is an increasing interest in physical activity and sport for adolescent girls and young adults worldwide. In adult women, exercise can contribute to disease prevention and management.

Keywords: Physical Activities; Sports; Female Pelvic Floor; Management
\end{abstract}

\section{Introduction}

During the last three decades a tremendous increase in female sports participation has been witnessed at all levels. The belief that urinary incontinence (UI) is mainly a problem of the elderly and parous women has been prevailing in our society [1]. Therefore, women who practice high-impact sports or who have higher volume of training should be aware of the symptoms associated with pelvic floor disorders (PFDs), since they seem to predispose to urine leakage. A large variety of sports may interfere with everyday life or training, leading athletes to compromise their performance or risk compromising it. This often-ignored problem alters an athlete's quality of life and sporting performance. The impact of this hot topic in women's health is rarely discussed within the context of sport. These women do not have the classic risk factors for PFDs including urinary incontinence (UI), pelvic organ prolapse (POP) such as age, parity and obesity. Sportswomen use strategies to minimize the loss of urine, like emptying the bladder before competitions, lowering the ingestion of liquids and restricting certain activities and avoiding some types of exercise that cause loss of urine, like jumping and running.

\section{Risk factors}

One of the most important medical problems that can occur with female athletes is the female athlete triad. It is a medical condition often observed in physically active and athletic females that involves the interrelationships among spectrums of energy availability, menstrual function and bone mineral density. All female athletes are at risk of the female athlete triad, regardless of body build or sport. This complex disorder was first coined by the American College of Sports Medicine in 1994 after many experts in the field had noticed a pattern among adolescent and young adult female athlete patient [2]. Nonetheless, the condition is most common in young female athletes in sports which focus on a thin and lean physique as is commonly seen in endurance sports, or in sports in which there is subjective judging. Low energy availability and inadequate caloric intake appear to be an underlying causative factor in the female athlete triad [2]. Eating disorders are associated with higher prevalence of both stress urinary incontinence (USI) and overactive bladder (OAB). In a study of Norwegian elite athlete [3] the prevalence of stress urinary incontinence (USI) was $49.5 \%$ and urgency urinary incontinence or overactive bladder (OAB) was $20 \%$ in eating disordered athletes. This was significantly higher than in healthy athletes. A significant correlation between UI, positive pad test and eating disorders was also reported in longdistance runners [4]. People who participate in long-distance running are among those athletes who are more vulnerable to developing an eating disorder. If other factors are involved, such as social pressures, low body image/self-esteem, or biological susceptibility, the risk for developing an eating disorder can be increased. Eating disorders may weaken the pelvic floor due to lack of energy and the nutritional factors needed to build and strengthen the muscles, ligaments and fascia. Several eating disorders involve induced vomiting, which generates repetitive high intra-abdominal pressure (IAP) on the pelvic floor muscles (PFMs), with further possible weakening. Considering that this problem can interfere with sports performance, it is necessary to pay special attention to this population, seeking to understand the factors triggering their urinary symptoms. Another issue is that women with POP or with UI have less collagen concentration in their skin and periurethral tissue, and a decreased ratio of type I to type III collagen. Hypermobility syndrome, which is characterized by a decreased ratio of type I to type III collagen, is associated with an increased prevalence of POP, but not UI [5]. Moreover, high-impact varsity athletes with increased foot arch flexibility had less UI, possibly because of the better shock-absorbing capacity of their lower extremities. Researchers acknowledge that, distal organs alignment can change pelvic girdle through powers imposed via feet. Different position's impact 
of feet on pelvic floor have been specified by some researchers. Individuals have maximum activity of PFMs, in standing position with dorsiflexion and have minimum rate of activity in position of plantar flection. This issue is on important factor combined with exercise therapy in USI. Nygaard., et al. [6] explore the relationship between UI in elite nulliparous athletes and force absorption on impact, as assessed by foot arch flexibility. There was a statistically significant association between decreased foot flexibility and UI, the mean percent change in arch height was $8.94+/-0.08 \%$ in incontinent women and $13.70+/-0.09 \%$ in continent women. How impact forces are absorbed may be one potential etiology for USI. An improved understanding of how impact forces are transmitted to the pelvic floor could provide important information about potential preventive interventions for UI and other PFDs, such as POP. Further studies are required to elucidate mechanisms for this interesting finding for re-education techniques. The aim of Pozo-Municio [7] was to determine the prevalence of genitourinary conditions in dance and to establish whether any correlation exists between SUI and the cushioning capacity of the foot. It is noteworthy that other systems can be affected by the demands of a disciplined physical exercise such as dance. A total of $68.7 \%$ of ballet and flamenco dancers had suffered a genitourinary condition at some time during their lives, and $48.1 \%$ of the female dancers. SUI was triggered by dance in $69.2 \%$ of the females and was considered as problematic by $30.7 \%$. SUI occurs during the classes in $55 \%$ of cases, mainly during ballet activities, followed by flamenco tap dancing. SUI associated with dance is more closely related to the flexibility of the plantar arch jumps and vibrations from foot tapping.

Links between compromised pelvic floor health and strenuous exercise are reported in the literature. Our knowledge of the PFMS of young female athletes is limited. Bo [8] described two opposing hypotheses about pelvic floor dysfunction associated with the practice of sports: (1): by strengthening the PFMs and decreasing the levator hiatus, exercise decreases the risk of PFDs, but negatively affects the ease and safety of childbirth; (2): by overloading and stretching the PFMs, exercise not only increases the risk of these disorders, but also makes labor and childbirth easier, as the PFMs do not obstruct the exit of the fetus. There are also at least three other hypotheses to explain the development of UI. In 1994, DeLancey., et al. [9] introduced the 'hammock' theory. From his cadaveric studies, he described the urethra as resting on a supportive layer of endopelvic fascia and anterior vaginal wall. DeLancey theorized that, when IAP is high, pressure is transmitted to the bladder neck and proximal urethra, resulting in urethral closure as it gets compressed against the rigid support of the pubocervical fascia and anterior vaginal wall. In 1990 Petros and Ulmstem [10] introduced the 'integral' theory of SUI and OAB. According to this theory, laxity of the anterior vaginal wall leads to activation of stretch receptors in the bladder neck and proximal urethra, resulting in detrusor overactivity. The laxity of the vaginal wall also leads to SUI because of a dissipation of urethral closure pressures. The "neurologic" hypothesis is associated with the pudendal nerve injury. The pudendal nerve can be compressed or stretched, re- sulting in temporary or permanent neuropathy. If the pelvic floor is over-stretched, acutely (e.g. prolonged or difficult childbirth) or chronically (e.g. chronic straining) the pudendal nerve is vulnerable to stretch-induced neuropathy. It has been suggested that the progressive denervation of the striated pelvic sphincter musculature that occurs in USI is due to repeated stretch injury of the innervation of these muscles when the pelvic floor diaphragm is weak. They concluded that it is very important to know about this neurogenic factor before considering rehabilitation. Pudendal nerve entrapment, also known as Alcock canal syndrome, is very rare and is associated with professional cycling. Possible causes of pudendal neuralgia include excessive bicycling (often known as the "cyclist syndrome".

Mild-to-moderate physical activity, such as walking, decreases the risk of UI but female athletes are about three times more likely to have UI compared to controls. There is some evidence that strenuous exercise may cause and worsen POP, but data are inconsistent. Little is known about how pelvic floor muscle structure or function differs in athletes. Assessed by MRI or by ultrasound, athletes showed a higher mean diameter of the pubovisceral muscle, greater bladder neck descent and a larger hiatal area on Valsalva maneuver. The type of sports played can have a major impact on the risk of developing PFDs and UI. In 1994, Bourcier and Juras [11] have developed a classification of risk to the pelvic floor musculature related to the level of impact of the sport. The classification described three groups of sport impact levels and risk related to the amount of pressure generated on the PFMs (Table 1). Perineal pressure is decreased in female athletes compared to non-athlete women. A lower perineal pressure correlates with increased symptoms of UI and PFDs. Using a novel wireless intra-vaginal pressure transducer, intra-abdominal pressure (IAP)was measured during diverse activities in a laboratory. There were statistically significant differences in maximal pressure between levels of walking, cycling and highpressure activities. The descriptive information provided by Shaw., et al. [12] may be especially relevant to educators and to those who provide exercise training for women in the context of fitness. Further research is needed to understand variability in IAP amongst women before instituting exercise guidelines and activity restriction based on IAP. There is a "continence threshold" which corresponds to the amount of time which the PFMs can withstand efforts and repetitive impacts. If this threshold is exceeded, these muscles are fatigued and lose their efficiency, especially if they have not been prepared for these conditions. Fatigue of PFMs might be the reason why women with symptoms of UI describe that leakage starts only after several incidents, such as repetitive jumps. This indicates that the continence control system weakens after repetitive stresses. In an ideal situation the regulation of pressure within the abdomen happens automatically: the muscles of the core work together in a good coordination. If any of the muscles of the core are weakened or damaged, this coordinated automatic action may be altered. If this occurs repeatedly over time the pelvic organs are strained. In fact, an increasing number of studies regarding UI in athletes indicate that it is not always associated with weak PFMs. Vaginal flatus is one of the other PFDs that have uncertain causes, which has re- 
ceived less attention. Different terms are described for Garrulitas Vulvae: vaginal wind, vaginal flatus, vaginal noise, or noisy vagina. Air emissions, or "queefs", from the vagina occur most commonly during sexual intercourse, certain physical activities such as gym and yoga as well as impairment in religious duty practice. It appears that other risk factors are linked to potential development of PFD in this group. In our Women's Sports Center, results show that the prevalence of vaginal flatus is $40 \%$. Increasing genital hiatus size is associated highly with vaginal support loss. If nulliparous female sportswomen have already congenital defects of Levator Ani associated with the risk factor of permanently repeated increases in IAP, they need to have mandatory screening.

\begin{tabular}{|l|c|}
\hline $\begin{array}{l}\text { Group I: High } \\
\text { impact sports }\end{array}$ & $\begin{array}{c}\text { Trampoline, skipping rope, volleyball, } \\
\text { handball, soccer, martial arts, weight lift- } \\
\text { ing, floor gymnastics, hurdles, waterskiing }\end{array}$ \\
\hline $\begin{array}{l}\text { Group II: Moder- } \\
\text { ate impact sports }\end{array}$ & $\begin{array}{c}\text { Horseback riding, tennis, badminton, } \\
\text { running, aerobics, snow skiing, trekking, } \\
\text { mountain biking, aquatic aerobics }\end{array}$ \\
\hline $\begin{array}{l}\text { Group III: Low } \\
\text { impact sports }\end{array}$ & $\begin{array}{c}\text { Nordic walking, swimming, rowing ma- } \\
\text { chine, kayaking, cycling, rollerblading, golf, } \\
\text { hiking, skating }\end{array}$ \\
\hline
\end{tabular}

Table 1

At the opposite side of the previous PFMs dysfunction, another issue is overactive PFMs also known as hypertonic PFMs or hypertonia. It is a condition where there may be increased PFMs tension at rest, increased voluntary or involuntary contractile activity, or a decreased ability to fully relax the PFMs, there is blood flow restricted and oxygenation reduced [13]. This restriction can create an acidic environment in the area. The PFMs can become so tense they are incapable of relaxing. It can also result in constipation, excessive tightness in the hips, piriformis, and hamstrings. The region's muscles and nerves begin deteriorating. The muscles become tender and the nerves begin to fire inappropriately, causing chronic pain. A hypertonic pelvic floor is a constantly contracted pelvic floor, this constant contraction puts the uterus, bladder, bowel and other structures under enormous amounts of pressure. Among different causes, some of them could be observed in young nulliparous athletes: joint hypermobility - with excessive hip and pelvic mobility can compensate through PFMs tightness; stresstension can be stored in the PFMs in response to stress; bladder or bowel dysfunction. Another topic is running, a high impact activity, particularly for women. Running hard, running downhill and sprinting all increase the pressure on the bladder, which may cause some leaking. Surprisingly, young, female runners, who had never been pregnant, were experiencing leakage with running. For many runners who hit the ground with poor technique, pelvic floor problems are worsened. The physical activity that is associated with landing passes through the lower limb and pelvic floor fast as the foot drops to the ground. Furthermore, overactive bladder (OAB) is a common condition during running and has a significant impact of quality of life on female recreational runners. Abitteboul Y., et al. [14] determine the prevalence of UI within a population of 800 female recreational runners during marathon. Among the participants, 268 (52.4\%) were marathon runners and 243 (47.5\%) were relay runners. Among responders, 157 (30.7\%) runners declared to UI symptoms (any circumstances). Among 157 runners who declared an UI, 83 (52,9\%) presented with urinary leaks during the running. In half of the cases, these urinary leaks usually arose at the end of race. In this series, the prevalence of UI was $30.7 \%$ within a population of female recreational runners. This rate seems to be similar to the current prevalence in the general population.

In conclusion on these different studies, data are insufficient to determine whether strenuous activity while young predisposes to PFDs later in life. The existing literature suggests that most physical activity does not harm the pelvic floor and does provide numerous health benefits for women. Prospective studies are needed in all populations, such as those with high genetic risk, LAM injury, and on women during potentially vulnerable life periods, such as the early postpartum or postoperative periods. Given how common both childbirth and surgery for PFDs are, it is surprising how very little data are present about how the dose and timing of physical impact the pelvic floor and subsequent PFMs [1].

\section{New treatment modalities}

Treatment can often help when symptoms are bothersome or restrict a woman's activities. The best approach to treatment of PFDs is multimodal. Urology, gynecology, gastroenterology, physical therapy, all can contribute their specialty expertise to address this patient who frequently had never asked help for her specific unusual problem related to physical activities and sports. Given the fact that UI as well as POP are not generally a progressive disease, conservative therapies play an integral part in the management. There is currently a variety of treatment options for female patients, ranging from conservative to surgical procedures. Among the different options offered, some may be proposed as being beneficial to female athletes as well. The different components of conservative management include behavioral therapy with lifestyle changes, pelvic floor muscle training (PFMT), biofeedback therapy (BF), electrical stimulation (ES) and mechanical devices. Whenever possible, surgeons use minimally invasive approaches to perform surgical procedures which are very rare in this type of young patients. If necessary and only in very rare cases, we may recommend a surgery called a mid-urethral sling which involves placing a small material (surgical mesh) under the urethra.

\section{Behavioral therapies}

Behavioral treatments improve bladder control by changing the incontinent patient's behavior, especially her voiding habits, and by teaching skills for preventing urine loss. These treatments are effective for most outpatient patients, urge or mixed incontinence. The advantages of behavioral interventions include the absence of side-effects, patient comfort and high levels of patient satisfaction. Although behavioral interventions are more accepted today than 20 years ago, they are still not widely available or integrated into most clinical practices, and we know little of how effective 
they will be in these settings. The aim of behavioral treatments is to improve bladder control by changing the incontinent patient's behavior, especially her voiding habits, and by teaching skills for preventing urine loss. Several types of behavioral methods are used for treating UI: bladder training, habit training, prompted voiding, and PFMT. The earliest form of behavioral treatment [15] for urge incontinence was the bladder drill, an intensive intervention that was usually conducted on an inpatient basis. Bladder drill procedures imposed a lengthened interval between voids to establish a normal frequency of urination and were reported to result in normalization of bladder function. Bladder training is a modification of bladder drill that is conducted in a more gradual fashion on an outpatient basis. Fluid restriction has also been recommended in the treatment of $\mathrm{OAB}$ as excessive fluid intake can exacerbate symptoms. Conversely, extreme fluid restriction produces concentrated urine, which has been postulated to be a bladder irritant, leading to frequency, urgency, and urinary tract infections. Female athletes with $\mathrm{OAB}$ should try moderately restricting fluid intake as applicable to their sport and health. Multicomponent behavioral training is another form of behavioral treatment that includes pelvic floor muscle rehabilitation and focuses less on voiding habits to prevent stress as well as urge accidents. PFMT and exercises were originally designed to teach patients how to control and exercise peri-urethral muscles, with the goal of using voluntary contraction of these muscles to occlude the urethra during physical activities that cause USI. Using biofeedback (BF) or other teaching methods, patients learn to contract PFMs selectively while relaxing other muscles, such as AbMs, that can increase pressure on the bladder. However, it has been demonstrated that voluntary PFMs contraction can also inhibit detrusor contraction [16]. This technique is now frequently used as a component in the treatment of urge incontinence as well. In addition to using pelvic floor muscles to occlude the urethra, patients learn to use pelvic floor muscle contraction and other urge suppression strategies to inhibit bladder contraction. Among the advantages of behavioral interventions are safety, absence of side effects, patient comfort, and high levels of patient satisfaction. In addition, nurses, nurse practitioners, and other health care providers who treat patients in outpatient office settings can implement the procedures successfully. Sports medicine physicians working with the athlete can help to monitor and suggest optimal regimes to avoid dehydration, while minimizing problems with urinary leakage [1].

\section{Pelvic floor muscles training (PFMT)}

PFMT is the most commonly used physical therapy treatment for SUI. It is sometimes also recommended for mixed UI and less commonly for OAB. PFMT should be the first line treatment as it is had no serious adverse effects, however, the training needs proper instruction and close follow-up to be effective. Although there is a plethora of treatment options, conservative management is the first-line option for most patients with UI. The rationale for conservative treatment is that UI is not necessarily a progressive disease, and that conservative therapies can be effective, well tolerated, and safe [17]. PFMT use began gaining popularity in the mid- $20^{\text {th }}$ cen- tury thanks to Arnold Kegel's success in treating women with SUI [18]. PFMT for treatment of SUI and OAB is thought to utilize PFMs in distinct ways: by increasing urethral pressure, through support of the bladder neck, and by interacting with the transversus abdominis (AbMs) via coordinated contractions between the pelvic floor muscles and the transversus abdominis. In the case of UI, it is thought that PFMs contraction can inhibit detrusor contractions. The PFMs consist of several muscles that include the anal sphincter and the Levator Ani muscles (LAMs), including: puborectalis, pubococcygeus, and iliococcygeus muscles. The normal function of the PFMs is to squeeze around the vaginal, urethral, and anal openings and to lift inwards in a cranial direction. The premise is that strong LAMs contraction will improve urethral closure and pelvic organ support. Since the LAMs consist of both type I (slow twitch) and type II (fast twitch) muscle fibers, specific strength training exercises can affect type II muscle fiber size through hypertrophy. Thus, strengthening type II fibers of the LAMs could aid in maintaining continence specifically. An optimal PFMs exercise regime would change the morphology and position of PFMs to enable subconscious contraction, a mechanism thought to occur in continent women. The principles of PFMT are overload, specificity, maintenance and reversibility. Overload can be achieved by maximum contractions, lengthening of the holding periods, increase in number of repetitions and reduced rest intervals. However, the exact methods used often vary and it is now recommended that PFMT should be performed in the following manner. Three sets of eight to 12 slow velocity maximal voluntary PFMs contractions sustained for six to eight seconds each. These should be performed three to four times per week and continued for at least 15 - 20 weeks. To improve strength and/or timing of the PFMs contraction will take a minimum of four to eight weeks. Maximal contractions and the duration of the exercise period are the most important factors in increasing and maintaining muscle strength. At least 15 - 20 weeks of exercise are recommended, because the effects in the first 6 - 8 weeks [19]. There is no standardized treatment program, but the National Institute for Clinical Excellence (NICE) recommends a trial of PFMs exercises, consisting of at least eight contractions three times a day for a minimum of 3 months, as a first-line treatment for UI. Long-term results depend on compliance and motivation. They are important factors for the success of PFMT, furthermore, appropriate training and supervision by a health care professional is considered essential. Studies have shown that successful results were maintained after 10 years, in two-thirds of the patients originally classified as successful and 33\% of the patients had surgery. However, only $8 \%$ had undergone surgery in the group originally being successful after training, whereas $62 \%$ had undergone surgery in the group initially dissatisfied with training [20].

\section{Core stability and postural control}

Another consideration is the role of core stability posture which have to be taken account in the physical therapy sessions. Stability of the trunk play roles in the individuals not only in maintaining an upright body posture, but also in helping to change positions 
when sitting, standing and walking. In sports performances, core strength is also very important to improve body balance and postural control in movements such as landing and contact. The concept of core stability probably emanated from Australian research into postural control in both healthy and chronic low back pain populations. They were interested in the role of the motor system how the nervous system organizes the appropriate responses to support the spine, give us the postural control to counteract gravity and balance while at the same time, also co-coordinating important functions such as breathing and continence. Much of their research involved studying the feedforward anticipatory role played by the IAP mechanism and important aspect of the antigravity postural control and spinal stabilization system. They studied the roles of various muscles contributing to a synergy of muscles responsible for generating IAP: transversus abdominis, the diaphragm, the PFMs and lumbar multifidus. Due to their contribution to modulation of IAP and stiffness of the sacroiliac joints, the PFMs have been argued to provide a contribution to control of the lumbar spine and pelvis. Furthermore, as IAP is modulated during respiration this is likely to be accompanied by changes in PFMs activity. Hodges., et al. [21] evaluate the postural and respiratory function of the PFMs, recordings of anal and vaginal electromyographic activity made with surface electrodes during single and repetitive arm movements that challenge the stability of the spine that challenge the stability of the spine. This study provides evidence that the PFMs contribute to both postural and respiratory functions. Smith., et al. [22] determine whether postural activity of the PFMs and AbMs differs between continent and incontinent women during rapid arm movements that present a postural challenge to the trunk. In many incontinent women, PFMs EMG decreased before the postural activation. These findings would be expected to have negative consequences for continence and lumbopelvic stability in women with incontinence. Investigating the activity of the PFMs in women during impact activities such as jumping, running or coughing Moser., et al. [23] may elucidate different aspects of PFMs activation and therefore clarify the pathophysiology of SUI. A systematic review was conducted to summarize current evidence on PFMs activity during impact activities in both continent and incontinent women. The findings of this systematic review suggest that impact activities causing involuntary and reflex PFMs activity should be the subject of further study. This may help guide clinical studies to improve our understanding of how the PFMs react during impact activities and to determine best practices that can be included in rehabilitation programs. The timing of PFMs activity in relation to the activity of other trunk muscles seems to be a crucial factor in maintaining continence. They suggest that this may help to determine best practices that can be included in rehabilitation programs. It is worth mentioning that health care professionals may also need to consider the body position in which the women with SUI exercise. Borello-France., et al. [24] compared the efficacy of a PFMs exercises progression that included practice in upright positions (i.e. sitting and standing) with the effectiveness of a PFMs exercises program that was performed only in the supine position in reducing SUI. Women in this study achieved a mean
$67.9 \%$ reduction in the frequency of SUI episodes and improvements in other study outcomes. They showed that the position used for PFMs exercises was not an important factor in performing exercise and the resulting reduction in SUI. Women in this study achieved a mean $67.9 \%$ reduction in the frequency of SUI episodes and improvements in other study outcomes. Clinically, specialists utilize exercise progression from gravity-eliminated to anti-gravity positions and finally an unstable base of support such as Swiss ball exercise when strengthening the PFMs. It is in the same direction we had when we proposed the MAB Program (see Biofeedback section). The PFMs need to be much stronger in elite athletes in certain sports than in other non-athletes. During low levels of activity, the fatigue-resistant slow twitch-fibers are predominately activated. When activity increases, fast-twitch fibers are also recruited. This highlights another difference between training athletes and training non-athletic women with UI. As the core is increasingly incorporated in yoga, Pilates and other techniques, PFMT can be taught. We recommend that PFMT be taught in a general exercise class with a former assessment of ability to perform a correct contraction [1]. It can then be taught in smaller classes to offer a wide range of programs for the beginners to the advanced participants. Rivalta., et al. [25] highlight another difference between training athletes and training non-athletic women with UI. As the core is increasingly incorporated in yoga, Pilates and other techniques, PFMT can be taught. They recommend that PFMT be taught in a general exercise class with a former assessment of ability to perform a correct contraction. It can then be taught in smaller classes to offer a wide range of programs for the beginners to the advanced participants.

\section{Biofeedback therapy (BF)}

PFMT with BF can be defined as the use of monitoring equipment to provide individuals with information on the functional activity of their PFM. Vaginal, anal sensors, surface EMG electrodes, intra-vaginal or anal balloons have been used to make patients more aware of muscle function, and to enhance and motivate patient efforts during training. A correct PFMs contraction was defined as an inward movement and squeeze around the urethra, vagina and rectum, but almost $25 \%-30 \%$ of women are unable to initially contract their PFMs correctly. These women contract the glutei, hip adductors, or quadriceps. Another common mistake is to produce a Valsalva maneuver. Instead of lifting up with PFMs, the patient is observed to be bearing down, which is counterproductive by increasing the intra-abdominal pressure. This response very common with sportswomen has been referred to as a "reversed perineal command" or a "paradoxical perineal command" [16]. In this group, the vast majority are sportswomen who have had the constant abdominal muscle recruitment involved in their physical activities. The prevalence of this improper contraction is more than $40 \%$ in our clinic and the co-activation of AbMs is about $50 \%[16,26]$. The first step in BF is to help the patient identify the proper muscles, how to recruit them and minimizing inappropriate recruitment of antagonist muscles. Therefore, abdominal muscle activity is a normal response to PFMs exercise in subjects with no 
symptoms of PFDs and provide preliminary evidence that specific abdominal exercises activate the PFMs. Increased co-contraction of the TrA may be associated with maximal contraction of PFMs in women without PFDs. During muscle contraction in PFMs, there is a rapid and stronger coactivity of the abdominal muscles. The stronger coactivity of these AbMs could cause an increase in intraabdominal pressure that, added to the insufficient PFMs contraction, would worsen the symptoms of PFD. Further research is needed to provide a better understanding of the co-contraction between the PFMs and AbMs [26]. Constantinou [27] evaluated the PFMs function from the dynamics of organ displacement produced during voluntary and reflex activation using technical innovations including ultrasound analysis of movement and MRI. A quick, strong and well-timed PFMs contraction can clamp the urethra, increasing the urethral pressure and prevent the leakage during an abrupt or sustained increase in IAP. On this basis, a process aims to demonstrate how the PFMs must be contracted before and during increased IAP was proposed. This technique is called the "perineal blockage before stress technique", the "stress strategy", the "Counter Bracing Technique" but most commonly the "Knack maneuver" $[1,26,28]$. The Knack is a strong and well-timed contraction of the PFMs. It involves the pelvic floor muscles contracting immediately before and during any increase in downward pressure on the pelvic floor. We may assume that erect posture and a constant increase in IAP during physical activities alter the urethra-vesical function. We can assume a certain relationship between pelvic floor support and IAP during sport practice. On the basis of this concept my colleagues and I developed the applied biofeedback technique referring to EMG pattern of the PFMs combined with synergic and antagonistic muscle activity in a standing position. We have implemented a new technology using a short vaginal sensor (a tampon as visual BF) and surface electrodes set for accessory muscles (transverse, obliques and glutei) during PFMs exercise program, using the perineal blockage or the Knack technique. We developed the "MAB Program" $[1,16,26,29]$ using sports equipment (rowing machine, treadmill, body training machine) which provides a muscular framework that protects internal organs by involving the pelvic floor and core musculature. This equipment represents the opportunity to combine strength, endurance and speed and must be taken into account during treatment sessions for sportswomen. This MAB Program allows the participant to perform real physical activities in a rehabilitative environment. Ambulatory telemetry systems allow patients to move around the gym, using small wireless portable monitor. This technique focuses on the measurement and analysis of biomedical signals using the telemetry technique with portable devices for exercising patients. Perineal ultrasound can be used to detect and quantify PFMs activity. Women who had previously been instructed in PFMT have been shown to be more likely to contract the PFMs properly. The real-time information provided by ultrasound imaging has been proposed as a possible source of $\mathrm{BF}$ that can be valuable during PFMs and AbMs. BF via ultrasound can be given to enhance understanding and improve pelvic floor function using "the Knack or perineal blockage" technique during coughing and straining [30]. Ultrasound imaging can provide real- time visual information about the direction of pelvic floor movement during a PFMs contraction, straining maneuver, or functional task (e.g. active straight-leg raise test). Ultrasound imaging is an excellent tool to assess pelvic floor function and dysfunction and is suitable as instrument for biofeedback-directed for assessment of PFMs function. It is a relatively new procedure and is recently can be incorporated as a form of BF during rehabilitation [31]. A new therapeutic option is home care BF system.

A growing number of BF devices and programs also are being marketed for home use, including: interactive computer programs or mobile devices. This process involves being connected to a device with sensors that provide $\mathrm{BF}$ about specific aspects of the body. In this type of BF, sensors are placed at various points on the body, close to the skin of the anus or with an intravaginal or anal probe connected to an electromyography or a pressure device. This technique that enables to visualize and measure voluntary contractions and the device assists women in strengthening or relaxing their PFMs. The proliferation of smart-phone devices offers a new platform for delivering mobile with gaming-style application that users can engage with in any setting. The sensors on the trainer record the contractions and transmit them via Bluetooth to the application. The new trend is to interface the home unit with an existing diagnostic system. The hand-held unit with a microcomputer provides a graphic display and can be connected to a clinic system, which record the progression of the pelvic floor program and conduct statistical analyses.

\section{Electrical stimulation (ES)}

ES is another potential option for management of UI in physically active and athletic women. Pelvic floor electrical stimulation activates pudendal nerve afferents, which in turn results in activation of pudendal and hypogastric nerve efferent, causing contraction of smooth and striated periurethral sphincter and PFMs. This provides a form of passive exercise with the goal of improving the urethral closure mechanism. In addition, ES can be useful in teaching PFMs contraction to women who cannot identify or contract these muscles voluntarily because of extreme weakness. There are many choices of electrode shape, size, and configuration to fit the need of the patient and therapeutic goal for ES. During ES, sticky pads or surface electrodes are stuck to the skin around the pelvic area or a vaginal sensor (probe) is placed into the vagina or rectum. Frequency settings between 20 and $50 \mathrm{~Hz}$ have been reported as optimal for sphincter closure and PFMs contraction, and 5 to $20 \mathrm{~Hz}$ for reflex detrusor inhibition [32,33]. It uses natural pathways and its efficacy relies on a preserved reflex arc, with complete or partial integrity of the PFMs innervation. The effect has been explained as a re-education or a reactivation of lost functions of the PFMs. The physiological objectives of ES are to produce muscle hypertrophy, to normalize the reflex activity of the lower urinary tract, and to increase circulation to muscles and the capillary system. ES of the pudendal nerve improves urethral closure by activating the PFMs. It may also increase conscious awareness of the action of these muscles to yield an improved ability to perform a voluntary mus- 
cle contraction [34]. The treatment is generally given in a healthcare professional's office. Lately small stimulation machines that deliver an electrical current are becoming more common making home use the goal. Neuromuscular electrical stimulation (NMES) is now available for patients unable to contract the muscle itself, as well as for those suffering from UI. Some stimulators are simplified and have preset stimulation settings (frequency, amplitude and duration) targeting different dysfunction. Home-based ES is as effective in the treatment of UI as outpatient clinic-based ES with significant improvement in objective and subjective parameters. Some electrodes are designed for placement in the vagina or in the anus, other electrodes are coated with conductive adhesive and attach to the skin of the pelvic floor. Correira., et al. [35] evaluate the effects of surface electrical stimulation and to compare them with the effects of the intravaginal electrical stimulation in women SUI. Both improved the QOL, urinary leakage, and strength and pressure of PFMs contraction. Another intravaginal device offers a new technology. The body responsive foam is soft, can be easily compressed to position inside the vagina and it adapts to fit each individual woman's shape, like a tampon. The device contains a microprocessor that delivers pulses and is automatically adjusts to the level of intensity upwards or downwards for a 30 minutes session [36]. A non-invasive electrical stimulation with no probe exists that can deliver NMES via a pair of lycra cycling shorts. Dmochowski., et al. [37] compare the efficacity and safety of this neuromuscular device with an FDA-approved intravaginal device for 12 weeks in a prospective, randomized, single-blind multicenter study. The NMES significantly improved objective and subjective measures of SUI, although statistical noninferiority was not established. The NMES was well tolerated and associated with fewer urinary tract infections than the comparator. The NMES provides a safe, clinically effective, conservative treatment option for female SUI and a low-risk alternative to intravaginal devices. Until recently ES was only possible through direct application or electrical current. However, new devices utilizing a pulsed electromagnetic field have been developed, which may achieve a similar affect. Extracorporeal magnetic innervation (ExMI) is a technology used for the treatment of UI. As a result, ExMI offers a noninvasive, effective and painless treatment. Further studies are needed to address how long the therapy will continue and benefits will last and whether retreatment or continuation therapy sessions will be necessary. In young nulliparous women, who are unable to isolate or adequately contract their PFMs, ExMI using a chair could be well accepted in this group and proposed before the other type of ES [38].

\section{Mechanical devices}

Mechanical devices are made of plastic or other materials. They are placed within the urethra or vagina in order to stop or control the leakage of urine. A very simple device which have the advantage for strengthening of the PFMs are vaginal weights or cones. Vaginal weights are cone-shaped and sold in sets of progressive increments. They are believed to promote correct PFMs contraction (through feedback sensed about cone slippage) and enhance PFMs strength (through progressive resistance training) [39]. As part of a progressive resistance program, women insert a weight intravaginally and are instructed to attempt to retain it by contracting the pelvic muscle for up to 30 minutes at least twice daily. In our practice we recommend our patients to use them while performs in upright position, physical activities because feedback occurs when the cone begins to slip downwards in the vagina. Weighted vaginal cones may be associated with PFMT or ES. Urethral occlusive devices may be inserted into the urethra or placed over the urethral meatus to prevent urinary leakage. These devices are palliative measures to prevent involuntary urine loss. Urinary control urethral insert devices have been introduced as a non-surgical remedy to treat female USI. A tampon-like devices come with a tamponstyle applicator. But, rather than a wad of absorbent fibers, they are collapsible silicone structures with a non-absorbent polypropylene covering. Once inserted into the vagina, the support expands to lift and support the urethra, helping to stop leaks from stresses such as coughing, sneezing or exercising. Although urethral inserts are potentially applicable to almost all women with pure SUI, the fact that these devices must be removed and reinserted with each void is not attractive to most women. The highest patient acceptance seems to be among those with very predictable, episodic SUI, such as during sports. An extensive range of mechanical devices have been described for the treatment of POP. Although primarily used for POP, there has been interest in developing devices specifically for SUI. Pessaries, which work by providing mechanical support for the urethra, have been used for the treatment of SUI. Some potential advantages of these vaginal support devices are that they can potentially be applicable to the majority of the incontinent population, they have mild side effects. Furthermore, these devices do not correct intrinsic sphincter deficiency, and may not help patients with hypermobility. Pessaries can be used to try to restore the prolapsed organs to their normal position and hence to relieve symptoms. Physicians generally offer pessaries for women with POP who desire nonsurgical management, future pregnancy, have early-stage POP. Additionally, pessaries are a valid option for patients with stress incontinence worsened by strenuous physical activity. There are currently many shapes and sizes of pessaries available to suit individual needs. Type of pessaries include: Ring, a circle-shaped device; Gehrung, a U-shaped pessary; Gellhorn, a disk-shaped device; Cube, a compacted down and inserted into the vagina. Ring pessaries are the most widely available and most commonly used. The Cube pessary works using suction to the vaginal walls are their sides are concave. It is becoming very popular PFMs and largely recommended by the midwives after childbirth. We believe that anyone wearing a pessary should also have a regular practice during the period of pessary treatment. Pelvic floor support belt can be used while exercising to prevent straining the PFMs. It can also be used while standing for long periods of time and lifting anything. It can help prevent the feeling that everything is going to fall out. Very few pelvic support belts for women with a POP exist on the market today and create a supportive, effective and comfortable pelvic brace. There is also support panty that help the woman to exercise when she has USI, POP and pelvic girdle pain. Sports shorts for weak are innovative running shorts designed for wom- 
en with weak PFMs, the shorts give support in the pelvic area to support PFMs. Other cheaper options are the use of a tight bicycle shorts as a perineal support or tampons to attempt to reduce their minor prolapse during physical activities. Pessaries are currently a conservative management tool and could be combined with PFMT.

\section{Surgical options}

For women who decline or have insufficient improvement following conservative therapy, there are a variety of surgical treatments. The introduction of midurethral slings (MUS) in 1996 [40] has changed the decision process for surgery. There are several procedures vary by the location of the sling and by the sling material: retropubic (TVT), transobturator (TOT), single-incision sling or mini-sling (SIMS) and adjustable midurethral sling. The Food and Drug Administration has issued public health warnings regarding the use of vaginal mesh kits for treating POP, which has created confusion and anxiety regarding the use of mesh for procedures, such as retropubic (TVT) and transobturator (TOT) slings. The FDA ban does not apply to midurethral slings for treating urinary incontinence. The extremely low complication rates that necessitated reoperation $3.2 \%$ due to problems with bladder emptying and $1.9 \%$ due to mesh exposure in the vagina should reassure providers and patients of the safety of MUS [41]. There is a significant body of research to support its use in sub-urethral polypropylene slings, including more than 15 years of experience in the USA and more than 20 years of experience in Europe. Recovery time differs from one person to another but we recommend that to limit activities for a full 6 weeks after surgery and to avoid any activity which will unduly increase the IAP. Healing takes around 3 months after POP repair but with MUS, the recovery is shorter less than one month. During this time patient should avoid any task that can put pressure on the repair i.e. lifting, straining, vigorous exercise, and constipation. Most patients return to full activity an average of three weeks after surgery. Therefore, the patient should abstain from physical activities for four to six weeks after surgery, depending on the type of surgery and the group of sports (low impact vs high impact). For sportswomen we have recently proposed a specific "post- surgical pelvic floor rehabilitation program" in order to reduce the risk of repeat surgery. After orthopedic or cardiac surgeries, post-surgical program of re-establishing joint motion, muscle strength joint function is always proposed for the patients. Physical therapy is a vital part of recovery after surgery. In the surgery in urogynecology, this is not the current practice. The second important point is that rehabilitation is a progressive activity. In this context, progression means safely increasing workloads and stimuli on the repaired tissue during the recovery process. Consequently, the core of rehabilitation is doing the right exercises at the right time, with the right balance between not enough and too much exercise. In our post- surgical pelvic floor rehabilitation program, with expert guidance, the exercises will be safer and more effective, improving the recovery. Additionally, rehab offers a personalized treatment plan shaped to the individual needs, including the type of surgery (MUS or POP repair) and any other medical conditions they may have $[29,42]$.
Laser and radiofrequency therapies

Due to various causes ranging from genetics, vaginal delivery, LAMs defects or even trauma, the vagina becomes loose and lax over time, resulting of vaginal flatus or vaginal noise. Air emissions, from the vagina occur most commonly during sexual intercourse, certain physical activities such as gym and yoga. Energy-based devices using radiofrequency (RF) and laser therapy (LT) have gained popularity as therapies for vaginal atrophy, UI, and POP. They have been promoted by cosmetic and aesthetic industries for vaginal "laxity" and vaginal "rejuvenation". The LT stimulates blood flow to the affected areas and improvement of the connective tissue and therefore of the firmness of the vagina. The study of Lang and Karram [43] concluded that currently, we are in the early stages of scientific investigation into the use of lasers in the treatment of PFDs, but the emerging data is encouraging. At the present time there is insufficient data to promote these therapies for USI, vaginal tightening, or other pelvic floor abnormalities. Another treatment modality for USI is a transvaginal (RF) applicator to deliver radio frequency energy to the endopelvic fascia. The purported mechanism of effect for this therapy is shrinkage of the collagenated tissue which composes the endopelvic fascia that supports the bladder neck and proximal urethra, thus stabilizing the proximal urethra and bladder neck. Several devices in the market currently offer RF-based vaginal rejuvenation. None of these technologies require anesthesia and on average, the treatment duration spans 15 to 30 minutes. Patients report feeling a sensation of warmth, which is very tolerable, and due to the lack of downtime, they can resume their normal activities the same day. A recent article of this topic aims to review the current available literature and its quality on this emerging technology [44]. This international multidisciplinary expert panel concluded that no randomized controlled trials have been published, no comparative studies to existing treatment have been carried out. This international group propose that whilst there remains a paucity of good quality data describing the safety, benefits, and appropriate use of vaginal radiofrequency or laser treatments a consensus best practice document by an established scientific community needs to be developed. The emergence of energy-based devices enables women and their physicians to have another treatment option in their armamentarium to consider, while weighing the associated risks and benefits. The latest MUS can be preformed in day surgery, with clear advantages compared to traditional procedures. A novel approach through the use of LT could represent an additional opportunity, an outpatient method to treat USI. However, long-term well-designed prospective studies are still needed to disclose the effectiveness of LT and others MUS in SUI.

\section{Conclusion}

PFDs are common in physically active women and female athletes, especially in the latter participating in moderate and high intensity exercise and sports. There is great need to document the acute and chronic effects of exercise and sport participation on the pelvic floor. Such information is important as expert consensus suggests that long-term, elevated IAP predisposes women 
to PFDs. Based on our long experience with the management of young female athletes we can attest that there are rewarding and promising treatment options for women suffering from PFDs. With the use of a complete pelvic floor rehabilitation program in female athletes affected by UI, we propose a combination of PFMT, pelvic core workout, BF, ES and sometimes mechanical devices (Table 2 and 3). This specific program could represent a good choice of treatment for such a population. It should be stated that exercise and sport promote good health-related outcomes and should be encouraged. We would recommend that women affected by these symptoms should seek medical help from a healthcare professional in this field. Sports medicine providers should become familiar with these PFDs and UI as well as the management options for physically active women and female athletes.

- $\quad$ Specific contraction of the pelvic muscles is the most direct treatment.

- $\quad$ Focus on quick twitch ability, sustained contraction and sub-maximal training.

- Watch for substitution patterns from abdominals/glutes/ adductors, or Valsalva.

- Work in multiple positions, with functional activities (squatting, standing).

- Add in pelvic floor synergistic muscles if participants are unable to coordinate the muscles.

- Avoid cross-over abdominals in clients with diastasis and emphasize transverse.

- $\quad$ Teach proper breathing patterns alone and in conjunction with PFMs activity.

- Use the postural education and core stability techniques.

- $\quad$ Possible use of vaginal cones during training.

- $\quad$ Possible use of pessary to support the pelvic organs during sports.

\section{Table 2}

- Avoid any sit-up exercises.

- Avoid holding plank positions for long duration.

- Decrease load in core exercises.

- Stay well hydrated to reduce constipation.

- Eat a high fiber diet to reduce constipation.

- Consider reducing your caffeine intake as caffeine can irritate the bladder.

- When performing pelvic training, advise athletes to watch out for the following:

- Heaviness/dragging/discomfort in the vaginal region.

- Bulging/doming/straining/discomfort in the abdominal region.

- Leaking of any sorts. ü Pelvic pain (pubic region, sides, or posteriorly) or lower back pain.

\section{Table 3}

\section{Bibliography}

1. Bourcier AP., et al. "Pelvic floor disorders in physically active women and female athletes". Leading Opinion Gynäkologie and Geburtshilf 3 (2018): 54-57.

2. Nattiv A., et al. "The Female Athlete Triad". Medicine and Science in Sports and Exercise 39.10 (2007): 1867-1882.

3. Bø K., et al. "Prevalence of stress and urge urinary incontinence in elite athletes and controls". Medicine and Science in Sports and Exercise 33.11 (2001): 1792-1802.

4. Araujo MP., et al. "The relationship between urinary incontinence and eating disorders in female long-distance runners". Revista da Associação Médica Brasileira 54 (2008): 146-149.

5. Norton PA., et al. "Genitourinary prolapse and joint hypermobility in women". Obstetrics and Gynecology 85 (1995): 225228.

6. Nygard IE., et al. "Relationship between foot flexibility and urinary incontinence in nulliparous varsity athletes". Obstetrics and Gynecology 87.6 (1996): 1049-1051.

7. Pozo-Municio C. "Genitourinary conditions in young dancers: relationship between urinary incontinence and foot flexibility". Journal of Dance Medicine and Science 11-2 (2007): 49.

8. Bø K. "Urinary incontinence, pelvic floor dysfunction, exercise and sport". Sports Medicine 34.7 (2004): 451-464.

9. DeLancey J. "Structural support of the urethra as it relates to stress urinary incontinence: the hammock hypothesis". American Journal of Obstetrics and Gynecology 170.6 (1994): 17201723.

10. Petros PE., et al. "An integral theory of female urinary incontinence. Experimental and clinical considerations". Acta Obstetricia et Gynecologica Scandinavica 153 (1990): 7-31.

11. Bourcier AP., et al. "Urinary incontinence in sports and fitness activities". Medicine and Science in Sports and Exercise 26.5 (1994): S14.

12. Shaw JM., et al. "Intra-abdominal pressures during activity in women using an intra-vaginal pressure transducer". Journal of Sports Sciences 32.12 (2014): 1176-1185.

13. Faubion SS., et al. "Recognition and Management of Nonrelaxing Pelvic Floor Dysfunction". Mayo Clinic Proceedings 87.2 (2012): 187-193.

14. Abitteboul Y., et al. "Urinary incontinence in non-professional female marathon runners". Progrès en Urologie 25.112 (2015): 636-641.

15. Frewen WK. "Role of bladder training in the treatment of the unstable bladder in the female". Urologic Clinics of North America 6 (1979): 273-277. 
16. Bourcier AP. "Behavioral Modification and Conservative Management of Overactive Bladder". In: Raz S, Rodriguez LV (eds): Female Urology, $3^{\text {rd }}$ edition. Elsevier Saunders, Philadelphia (2008): 213-233.

17. Moore K., et al. "Adult conservative management". In: Abrams P, Cardozo I, Khoury S, Wein A (eds). $5^{\text {th }}$ International Consultat on Incontinence. Heath Public Ltd Paris (2013).

18. Kegel A. "Progressive resistance exercise in the functional restoration of the perineal muscles". American Journal of Obstetrics and Gynecology 56.2 (1948): 238-248 .

19. Bø K. "Pelvic floor muscle training is effective in treatment of female stress urinary incontinence, but how does it work?". International Urogynecology Journal and Pelvic Floor Dysfunction 15 (2004): 76-84.

20. Cammu H., et al. "A 10-year follow-up after Kegel pelvic floor muscle exercises for genuine stress incontinence". BJU International 85 (2004): 655-658.

21. Hodges PW., et al. "Postural and respiratory functions of the pelvic floor muscles". Neurourology and Urodynamics 26 (2007): 362-371.

22. Smith MD., et al. "Postural activity of the pelvic floor muscles is delayed during rapid arm movements in women with stress urinary incontinence". The International Urogynecology Journal 18 (2007): 901-911.

23. Moser H., et al. "Pelvic floor muscle activity during impact loads in continent and incontinent women: a systematic review". The International Urogynecology Journal 29.2 (2018): 179-196.

24. Borello-France DF., et al. "Effect of Pelvic-Floor Muscle Exercise Position on Continence and Quality-of-Life Outcomes in Women with Stress Urinary Incontinence". Physical Therapy 86.7 (2007): 974-986.

25. Rivalta M., et al. "Urinary incontinence and sport: first and preliminary experience with a combined pelvic floor rehabilitation program in three female athletes". Health Care for Women International 31.5 (2010): 435-443.

26. Bourcier AP., et al. "Urinary incontinence in physically active and sportswomen". In Appell RA, Bourcier AP, La Torre P (eds): Pelvic Floor Dysfunction: Investigations and Conservative Treatment. Rome, Casa Edit Intern (1992): 9-17.

27. Constantinou CE., et al. "Spatial-distribution and timing of transmitted and reflexly generated urethral pressures in healthy women". The Journal of Urology 127 (1982): 964-969.

28. Miller JM., et al. "A pelvic muscle precontraction can reduce cough-related urine loss in selected women with mild SUI". Journal of the American Geriatrics Society 46 (1998): 870-874.
29. Bourcier AP., et al. "Incontinence urinaire chez les sportives". Rev Genesis 199 (2018): 4-8.

30. Dietz HP., et al. "The use of perineal ultrasound to quantify levator activity and teach pelvic floor muscles exercises". International Urogynecology Journal and Pelvic Floor Dysfunction 12 (2001): 166-168.

31. Baessler K., et al. "Ultrasound Imaging”. In Baessler K, Schüssler B, Burgio KL, Moore KH, Norton P, Stanton SL (eds): Pelvic Floor Re-education London, Springer Verlag (2008): 135-143.

32. Fall M., et al. "Electrical stimulation. A physiological approach to the treatment of urinary incontinence". Urologic Clinics of North America 18 (1991): 393-407.

33. Plevnik S., et al. "Short-term electrical stimulation: home treatment for urinary incontinence". The World Journal of Urology 4 (1986): 24-22.

34. Bourcier AP. "Pelvic Floor Rehabilitation in the Management of Urinary Stress Incontinence". In Raz S, Rodriguez LV (eds): Female Urology, $3^{\text {rd }}$ edition. Philadelphia, Elsevier Saunders (2008): 310-327.

35. Correia GN., et al. "Effects of surface and intravaginal electrical stimulation in the treatment of women with stress urinary incontinence: randomized controlled trial". The European Journal of Obstetrics and Gynecology and Reproductive Biology 173 (2014): 113-118.

36. Oldham J., et al. "Evaluation of a new disposable 'tampon-like' electrostimulation technology (Pelviva") for the treatment of urinary incontinence in women: a 12-week single blind randomized controlled trial". Neurourology and Urodynamics 32.5 (2013): 460-466.

37. Dmochowski R., et al. "External electrical stimulation compared with intravaginal electrical stimulation for the treatment of stress urinary incontinence in women: A randomized controlled noninferiority trial". Neurourology and Urodynamics 38.7 (2019): 1834-1843.

38. Yokoyama T., et al. "Extracorporeal magnetic innervation treatment for urinary incontinence". International Journal of Urology 11.8 (2004): 602-606.

39. Laycock J., et al. "Pelvic floor reeducation for stress incontinence: comparing three methods". British Journal of Community Nursing 6.5 (2001): 230-237.

40. Ulmsten U., et al. "An ambulatory surgical procedure under local anesthesia for treatment of female urinary incontinence". International Urogynecology Journal and Pelvic Floor Dysfunction 7.2 (1996): 81-85.

41. Kobaschi KC., et al. "Surgical Treatment of Female Stress Urinary Incontinence: AUA/SUFU Guideline". The Journal of Urology 198.4. (2017): 875-883. 
42. Bourcier AP. "Pelvic Floor Disorders in Female Athletes: General Concepts of Management". In $6^{\text {th }}$ annual meeting of the Congress European College of Sports and Exercise Physicians". Abstract book (2019).

43. Lang P., et al. "Lasers for pelvic floor dysfunctions: is there evidence?". Current Opinion in Obstetrics and Gynecology 29.5 (2017): 354-358.

44. Digesu GA., et al. "The energy-based devices for vaginal rejuvenation, urinary incontinence, vaginal cosmetic procedures, and other vulvo-vaginal disorders. An international multidisciplinary expert panel opinion". Neurourology and Urodynamics 38.3 (2019): 1005-1008.

\section{Assets from publication with us}

- Prompt Acknowledgement after receiving the article

- Thorough Double blinded peer review

- Rapid Publication

- Issue of Publication Certificate

- High visibility of your Published work

Website: https://www.actascientific.com/

Submit Article: https://www.actascientific.com/submission.php Email us: editor@actascientific.com

Contact us: +919182824667 